A puntes del CENES

ISSN 0120-3053

Volumen 30 - №. 52

Segundo Semestre 2011

Págs. 37 - 58

\title{
La estructura óptima de capital con reclamos contingentes: estudio de un caso en Colombia
}

\section{The optimal structure of capital with contingent claims: a case study in Colombia}

María del Pilar Báez Roa*

Juan Sergio Cruz M erchán**

Fecha de recepción: 28 de marzo de 2011

Fecha de aprobación: 30 mayo de 2011

* Administrador Industrial, especialista en Finanzas, especialista en Ingeniería de Software, magíster en Administración. Docente Escuela de Administración Industrial. Universidad Pedagógica y Tecnológica de Colombia. Correo electrónico: mariabaez11@gmail.com

** Administrador de Empresas, magíster en Política Internacional, profesor en varias maestrías y gerente de la Banca de Inversión Andes Riskgroup, Bogotá. Correo electrónico: juacruz2009@gmail.com 


\section{Resumen}

Uno de los debates de la investigación de la economía financiera, se relaciona con la existencia o no de una estructura óptima de capital que aporte valor a la firma. Al respecto, varios autores han estudiado el tema sin que aún sea posible un consenso. Se ilustra, desde la línea de las teorías que siguen la existencia de una estructura óptima de capital, un modelo que la determine utilizando los últimos desarrollos de valoración de la deuda, como lo son: la teoría de reclamos contingentes, basada en la teoría de opciones, para lo cual se sirve de información de una empresa colombiana.

Palabras clave: estructura de capital óptima, deuda, teoría de opciones, reclamos contingentes.

Clasificación JEL:D20, H74, C40, G10

\section{Abstract}

One of the debates of the research of the Financial Economics relates to the existence or not of an ideal structure of capital that contributes value to the company. About the matter, several authors have studied the topic but without a consensus. It is illustrated, from the theories that follow the existence of an optimal capital structure, a model that determines it using the last developments in valuation of debt, such as the theory of contingent claims, based on the theory of options, for which uses information from a Colombian company.

Keywords: optimal capital structure, debt, theory of options, contingent claims.

JEL Clasification: D20, H74, C40, G10 


\section{Introducción}

Uno de los puntos de gravedad de la investigación de la economía financiera se relaciona con la tríada: política de inversión, política de financiamiento o estructura de capital y valor de una empresa. El objeto concreto de esta triple relación es la pregunta si tiene sentido económico hablar de óptimo y bajo qué condiciones, restricciones y supuestos dentro de una concepción de la teoría económica neoclásica. Este tema fue dirigido hasta 1957 por investigaciones guiadas por Graham y Dood (1940); Durand (1952); y Dougall y Gutman (1955), citados por Rivera (2002), quienes consideraban que mediante una combinación de recursos propios y deuda se llega a un punto donde se minimiza el costo de capital y se maximiza el valor de la empresa, siendo esta la estructura óptima de la firma.
No obstante, este quietismo académico se ve interrumpido hasta el día de hoy, por las afirmaciones de Modigliani y Miller (en adelante MM), en sus famosos artículos

The cost of capital, $H$. Miller corporation finance, The theory of investment (1958); The cost of capital, corporation finance, The theory of investment: reply 1959; Corporate income taxes $y$ The cost of capital: a correction. En el ámbito de los mercados imperfectos surgen nuevas teorías entre las que encontramos la teoría de MM (1963), la irrelevancia de Miller (1977), Trade-off y la teoría de la jerarquía de las preferencias en las que se consideran diferentes imperfecciones como los impuestos, asimetrías de información, costos de dificultades financieras y costos de agencias, entre otros. 
Antes de analizarse ese artículo, es necesario hacer los siguientes comentarios. Es muy usual que los textos de finanzas corporativas presenten una síntesis del modelo analítico de estos dos últimos autores, desconociendo el proceso y la dinámica de la construcción del pensamiento y la obra de estos dos grandes maestros. La tensión entre diferentes escuelas económicas y en concreto, la tensión entre la visión clásica liderada por Durand y los autores seguidores del mismo, lleva a MM a dar una respuesta a las observaciones presentadas por el profesor Durand, en un artículo que se conoce como The cost of capital, corporation finance, and The theory of investment: reply 1959. Además, en el afán de sintetizar, se borran las fronteras muy precisas de los supuestos y los modelos en cada etapa. La anterior observación tiene un alto sentido para comprender el propósito de la investigación que nos ocupa, ya que la discusión ha girado y gira en torno a las implicaciones de las afirmaciones de nuestros grandes maestros en condiciones de competencia no perfecta $^{1}$. Por lo que el presente estudio pretende ilustrar desde la línea de las teorías que siguen la existencia de una estructura óptima de capital, un modelo que la determine utilizando los últimos desarrollos de valoración de la deuda, como la teoría de reclamos contingentes basada en la teoría de opciones, para lo cual se sirve de información de una empresa colombiana.
Para el desarrollo de este estudio inicialmente se revisan las principales teorías acerca de la estructura de capital, tanto en mercados perfectos como en mercados no perfectos; se caracteriza la metodología de reclamos contingentes basada en la teoría de opciones; se presenta la empresa en su aspecto financiero, para luego determinar la estructura óptima de la empresa Hilanderías Bogotá S.A. y finalmente se exponen las conclusiones.

\section{Teorías sobre la estructura de capital}

\subsection{Proposición I Modigliani Miller 1958 ( $\sin$ impuestos corporativos)}

Modigliani y Miller en su primera proposición determinan que el valor de una empresa depende del valor esperado de los flujos de caja proveniente de la operación descontada a una tasa que refleja el tipo de riesgo de clase del activo k. Los cinco supuestos centrales de esta definición son: no hay costos de transacción, no hay impuestos; las utilidades antes de intereses e impuestos esperadas $\mathrm{E}(\mathrm{X})$, de las distribuciones de probabilidad de todos los inversores de una firma son las mismas y permanecen constantes a lo largo del tiempo; todas las empresas pueden agruparse por clases homogéneas de rendimiento con igual o similar riesgo económico siendo perfectamente sustituibles las acciones de

\footnotetext{
Se podrían sintetizar las imperfecciones de mercado en este tema, así: impuestos corporativos y personales; costos por stress financiero o apuro financiero; asimetría de información; y teoría control de la empresa, etc.
} 
diferentes firmas que pertenecen a una misma clase de riesgo. También se supone una ausencia del riesgo en la deuda. Así definido el valor de la empresa como $\mathrm{V}=\mathrm{f}(\mathrm{NOI}, \mathrm{K})$, se establece la independencia del valor de la empresa de la arquitectura de las fuentes de financiación o estructura de capital. De allí se derivan fuertes conclusiones que han sido el epicentro del debate y de las investigaciones sin entrar a profundizar las implicaciones de los supuestos.

En primer término se independiza la política de inversiones de la forma como la operación se financia, ya que esta última es la que determina el valor de la empresa. La teoría post moderna de las finanzas claramente se alinea con la concepción presentada por MM, no obstante, ha avanzado de manera muy importante precisamente en demostrar la conexión entre las políticas financieras (lado derecho del balance) y las decisiones de inversión lado izquierdo del balance). En segundo término, al independizar el valor de la empresa de la estructura de capital no tendría sentido económico la gerencia financiera de los instrumentos financieros. Aquí es donde la ruptura de los supuestos de la primera proposición de MM aporta la ruta explicativa de la razón de ser de la gerencia financiera de riesgo. En tercer término, también los autores suponen que el costo de capital es independiente de la estructura de costo de capital y es una constante $\mathrm{K}$.

Posteriormente estos autores desarrollan una segunda tesis donde introducen el apalancamiento financiero determinando que el rendimiento esperado sobre las acciones de una firma apalancada aumenta linealmente con relación a la proporción $\mathrm{D} / \mathrm{E}$ definiéndose una prima por el riesgo del apalancamiento en que se incurre. Finalmente definen una tercera proposición en la cual argumentan que la forma de financiación de un proyecto no determina si es o no viable, sin embargo no por esto deja de existir una preferencia por la forma de financiarlo.

\subsection{Proposición I Modigliani y Miller 1963 (con Impuestos Corporativos)}

En esta proposición, los autores consideran el efecto de los impuestos en la estructura de capital, con la cual corrigieron su proposición I reconociendo las grandes ventajas tributarias en el valor de la empresa, así:

En una empresa no apalancada, el flujo de efectivo (UAII) después de impuestos es:

$$
U A I I=(1-\mathrm{T} c)
$$

En una empresa apalancada, el flujo de efectivo después de impuestos es:

$$
U A I I=\left(1-\mathrm{T}_{c}\right)+\mathrm{T}_{c} \mathrm{k}_{d}
$$

Ahora, el valor de una empresa no apalancada, es decir financiada totalmente con recursos propios, es el valor presente de sus flujos de efectivo descontado al costo de su capital social: 


$$
\mathrm{V}_{\mathrm{u}}=\frac{U A I I(1-\mathrm{T} c)}{\mathrm{k}_{\mathrm{o}}}
$$

donde:

$\mathrm{V}_{\mathrm{u}}=$ valor presente de una empresa no apalancada

UAII $=$ utilidades antes de intereses e impuestos

$\mathrm{Tc}=$ tasa fiscal corporativa

$\mathrm{k}_{\mathrm{o}}=$ costo de capital de una empresa totalmente financiada con recursos propios.

$\mathrm{k}_{\mathrm{d}}=$ costo de la deuda

$\mathrm{D}=$ deuda

Para determinar el valor de una empresa apalancada $\left(\mathrm{V}_{\mathrm{L}}\right)$ es necesario adicionar el valor presente de la protección fiscal proveniente de la deuda

$$
\begin{aligned}
& \mathrm{V}_{\mathrm{L}}=\frac{\operatorname{UAII}\left(1-\mathrm{T}_{c}\right)}{\mathrm{k}_{\mathrm{o}}}+\frac{\mathrm{T}_{c} \mathrm{k}_{d} \mathrm{D}}{\mathrm{k}_{d}} \\
& \mathrm{~V}_{\mathrm{L}}=\mathrm{V}_{\mathrm{U}}+\mathrm{T}_{c} \mathrm{~B}
\end{aligned}
$$

Como podemos observar, el mayor valor de una empresa apalancada se encontraría cuando está financiada totalmente con deuda. Sin embargo, en la vida real las personas no se pueden endeudar en un $100 \%$, ya que esta situación daría señales negativas al mercado y generaría unos costos de quiebra.

\subsection{Teoría del trade off o de equilibrio de la estructura de capital}

La teoría del trade-off comprende todos los modelos teóricos que propenden por una estructura óptima de capital que combine las fuentes de financiación de deuda y capital, donde maximiza el valor de la firma una vez se equilibren los beneficios y los costos de la deuda.Un incremento de deuda después de esta combinación, puede aumentar el riesgo, ya que la firma no podría cumplir con el servicio de la deuda e incrementaría el riesgo de los acreedores aumentando de esta forma sus dificultades financieras.

Para Fama y French (2002, p. 1), "los beneficios de la deuda incluyen la deducibilidad fiscal de los intereses y la reducción de la liquidez sin problemas, los costos de la deuda incluyen los posibles costos de quiebra y los conflictos de agencia entre accionistas y tenedores de bonos".

A continuación citaremos diversos estudios e investigaciones que encuentran relación con los lineamientos del tradeoff considerando diferentes determinantes en las decisiones de deuda-capital de las firmas. Wessles y Titman (1988, p. 17) evidenciaron que "los niveles de deuda están relacionados negativamente con firmas de una sola línea de negocios", por lo que su estructura óptima estaría en un nivel bajo de proporciones de deuda. Jensen y Meckling (1976), identifican dos tipos de conflictos: uno entre accionistas $\mathrm{y}$ administradores, $\mathrm{y}$ otro entre accionistas y prestamistas. los cuales hacen que se incurra en unos costos de agencia por lo que los mismos autores creen que la estructura óptima de capital podría ser obtenida por el intercambio de costos de agencia derivados de la deuda y de sus 
beneficios. Gilson (1997) en su estudio evidencia que los costos de transacción pueden tener un efecto en la elección de la estructura de capital de las empresas con dificultades financieras.

Fama y French (2002, p. 1) confirman unos de los lineamientos del trade-off: "las empresas con más inversiones tienen menos apalancamiento en el mercado". La estructura óptima de capital en Stulz (1990) está determinada por un balance entre la ganancia de la deuda en inversión preventiva en proyectos de valor decreciente contra el costo de la deuda en inversión preventiva en proyectos de valor creciente. Harris y Raviv (1991) argumentan que un alto nivel de deuda puede estar asociado a un mayor valor de la firma, siendo un mayor nivel de deuda relacionado con unos ingresos esperados y una baja probabilidad de reorganización en caso de un impago. DeAngelo y Masulis (1980) desarrollan un modelo que sigue el beneficio marginal de la deducción de impuestos corporativos por intereses, que varían con el apalancamiento y así producen un óptimo interior para aprovechar ${ }^{2}$.

\subsection{Teoría de jerarquía de preferencias (pecking order)}

Entre las teorías más destacadas que contemplan las imperfecciones del mercado tenemos la teoría de la jerarquía de las preferencias de Myers y Majluf, (1984), quienes desarrollaron un modelo de financiación e inversión bajo el supuesto que los administradores tienen información privilegiada de la empresa que los inversores externos no tienen y que estos inversores tienen decisiones racionales. Las principales características de este modelo son: para las empresas es mejor emitir valores seguros que con riesgo, es decir, en caso de necesitar una financiación externa se seleccionaría la deuda y no la emisión de acciones. Además, existe la posibilidad de rechazar una inversión si se han agotado sus emisiones de bajo riesgo en lugar de emitir con riesgo.

Las empresas para autofinanciarse pueden restringir el pago de dividendos en caso de bajas inversiones o emitir acciones cuando la asimetría de información es baja, pudiéndose decir que existe una alta correlación entre la política de dividendos y la información del valor de los activos por parte de los directivos. Asimismo, el modelo plantea que si los directivos tienen información privilegiada cuando se emiten acciones para financiar las inversiones, la cotización de las acciones disminuirá, pero si la empresa emite deuda sin riesgo no tendría disminución. Como podemos observar, las decisiones de financiación responden principalmente a la capacidad de generación interna de recursos; pero dado el déficit de estos recursos, se recurre a financiación externa, prioritariamente a la emisión de deuda, y si no se tuviera esta disponibilidad se

\footnotetext{
2 En su modelo óptimo, los escudos fiscales son diferentes a la deuda.
} 
renunciaría a desarrollar inversiones con VPN positivo (subinversión ${ }^{3}$ ) en lugar de la emisión de acciones. Esto nos indica que el modelo de la jerarquía de las preferencias no busca un nivel endeudamiento óptimo.

\subsubsection{Estudios sobre estructura de capital en Colombia}

Sarmiento (2005, p. 166), en su estudio realizado por medio de un modelo econométrico y de regresiones de las empresas colombianas del sector real determina que "con respecto al Flujo de Caja (1ra y 2da regresión) se obtiene una relación negativa y significativa entre esta variable y el nivel de deuda, es decir cuando se tienen recursos internos representados en flujos de caja se evita acceder a deuda. Con base en estos resultados, se estaría confirmando la teoría de la jerarquía de las preferencias para el caso colombiano, donde las firmas se financian principalmente con recursos internos al no tener acceso a la financiación externa para evitar problemas de infrainversión". Además, en el anterior estudio, se evidenció que a mayores problemas de asimetría de información, las firmas recurren con menor propensión a la financiación externa -en este caso deuda- pudiéndose presentar un problema de infrainversión, cumpliéndose uno de los lineamientos expuestos por Myers y Majluf (1984). Según este estudio, se considera la asimetría de información un determinante importante en las decisiones de financiación. Asimismo, los costos de transacción son otro determinante importante, ya que las empresas del sector real que cotizan en bolsa enfrentan costos de transacción cuando ajustan sus niveles de deuda.

Tenjo, López y Samudio en su estudio Determinantes de la estructura de capital de las empresas colombianas (19962002),

sugieren que un factor que marca de manera importante las decisiones de financiamiento de las firmas en el país es la existencia de imperfecciones en los mercados de recursos, derivadas de problemas de información imperfecta. La relación inversa que se encontró entre la rentabilidad y el coeficiente de endeudamiento de las firmas, uno de los hallazgos más claros del trabajo y base para esta afirmación, es el elemento central dentro de la literatura conocida como orden de prioridades. Los problemas de información imperfecta se manifiestan también en otras características de los mercados financieros en el país, tales como la concentración de crédito, la escasa disponibilidad de financiamiento de largo plazo y el incipiente desarrollo del mercado accionario, entre otros. Información asimétrica es, en pocas palabras, sinónimo de presencia de restricciones financieras en los mercados de recursos de financiamiento (2006, p. 32).

$\overline{3}$ No invertir en proyectos con VPN positivos. 
Chacón, Arroyo y Villalba, afirman:

que las PYME'S de Bucaramanga carecen de una planeación adecuada para definir su estructura financiera, el elevado tradicionalismo y empirismo predominan en el manejo de las organizaciones... Se presenta una imposibilidad de mercado y psicológica de los empresarios de acceder a fuentes de financiación "justas" de tal manera que empiezan a adquirir recursos a tasas elevadas, lo que conlleva a un aumento en el costo de capital que dificulta el cumplimiento del objetivo básico financiero... Los niveles de deuda que tienen actualmente las PYME'S se encuentran significativamente bajos respecto al óptimo arrojado por el modelo (2007, p. 16).

Como podemos concluir en este estudio, las decisiones de financiación de los empresarios de las PYMES en Bucaramanga no se enmarcan de manera muy clara dentro de ninguna teoría de estructura de capital anteriormente expuesta.

Rivera, en su estudio Estructura financiera y factores determinantes de la estructura de capital de las pymes del sector de confecciones del Valle del Cauca en el periodo 2000-2004, utilizando un modelo econométrico determinó que

La retención de utilidades tiene una relación negativa con el endeuda- miento de las pymes y las medianas empresas, lo cual se ajusta a lo establecido por la teoría de peckingorder: cuanto más retención de utilidades (recursos propios internos), menor endeudamiento". La relación negativa entre la rentabilidad y el endeudamiento a largo plazo de la pequeña empresa tiene su fundamento en la teoría del pecking-order, puesto que en escenarios de información asimétrica entre directivos e inversionistas extremos, las compañías prefieren usar recursos internos que le generan menos costos de información. Se supone que una mayor rentabilidad genera más fondos internos, lo que reemplazaría la opción de obtener fondos mediante deuda (2007, p. 211-212).

Cruz y Wadnipar, en su estudio Análisis de las variables y modelos que intervienen en la determinación de la estructura de capital de las empresas colombianas, mediante el modelo de la variable déficit utilizado por Frank y Goyal se obtuvo que

el aumento en la tangibilidad de los activos es inversamente proporcional al cambio en el nivel de deuda, lo cual se ajusta a la teoría de Pecking Order. $\mathrm{Al}$ tener mayor número de activos tangibles se disminuyen los problemas originados por asimetrías de información, originando que no sea subvalorada la empresa”. Otra característica muy importante a resaltar, es que la teoría de Pecking Order se 
ajusta en un nivel semifuerte, ya que la variable déficit no es la única que explica el cambio en el nivel de deuda adquirida, sino también las oportunidades de crecimiento, tamaño de la empresa y tangibilidad de los activos" (2008, p. 17).

De tal manera que las evidencias empíricas presentadas por dicho estudio respecto a la definición de la racionalidad del accionista y el criterio de selección que utiliza para definir la estructura de capital, en el sector de fabricación de telas y actividades relacionadas entre otros, es la existencia de un modelo de Pecking Order o jerarquización de las fuentes de financiación en un nivel semifuerte.

\subsection{Teoría de reclamos contingentes (CCA)}

La teoría inicial de los reclamos contingentes se focalizó en el optionpricing y la aplicación de la teoría de las opciones al análisis de la estructura del capital corporativo. El valor total de una compañía está dado por la suma del valor de los securities que esta tenga en su estructura de capital. Estos securities pueden ser vistos como reclamos contingentes del valor implícito de la compañía. El CCA puede ser implementado para analizar cómo cambia el valor de los reclamos contingentes a medida que cambia el valor de la compañía a lo largo del tiempo. Por lo anterior, este análisis de reclamos contingentes debe ser visto como una generalización de la teoría option-pricing con el fin de explicar el preámbulo en el cual todos los reclamos contingentes pueden ser valorados.

El análisis de reclamos contingentes se basa en tres principios: 1. El valor de los flujos del pasivo desde los activos. 2. Los pasivos tienen madurez diferente y por ende los riesgos relacionados son diferentes de acuerdo con su madurez y 3. Existe un elemento aleatorio respecto a la forma como el valor del activo evoluciona durante el tiempo. La deuda es un reclamador antiguo del valor de los activos y el patrimonio es un reclamador junior del valor del activo. La deuda es riesgosa porque el valor del activo puede no ser suficiente para cubrir los pagos de deuda contraídos. Debido a esto, el valor de la deuda riesgosa puede ser vista con dos componentes: el valor libre de probabilidad de no pago y la pérdida esperada asociada con el no pago, cuando los activos son insuficientes para cubrir los pagos de deuda contraídos. El valor de los reclamos junior (patrimonio en el caso de las compañías) se deriva del valor residual después de que se hayan hecho las promesas de pago de deuda.

Si el valor de los activos tiene un componente variable (por ejemplo: el precio cambia, shocks y otros factores que afectan el valor de los activos), entre más alta sea la volatilidad de los activos existe más probabilidad de que el valor de los activos caiga por debajo del nivel necesario para cubrir los pagos de las deudas maduras durante el periodo 
horizonte. En consecuencia, Ceteris Paribus, una mayor volatilidad implica tener una pérdida esperada mucho más alta y un menor valor de deuda riesgosa. Las técnicas financieras option pricing relationships han sido desarrolladas para medir la pérdida esperada como función del valor de los activos, de su volatilidad, la deuda libre de no pago (default), y el tiempo de horizonte. De la misma forma, el valor del patrimonio y de los reclamos junior pueden ser medidos en función de las mismas variables. La pérdida esperada en la deuda riesgosa es una opción put implícita. El patrimonio y los reclamos junior son opciones call implícitas.

La esencia del CCA son los cambios en las variables observadas - el valor de los securities en la estructura de capital - son usados para inferir cambios en las variables no observadas - el valor de la compañía -. La aplicación del CCA a la estructura de capital deriva de los pasivos maduros en la estructura de capital y en la identidad del balance en que el valor del mercado de toda la deuda más el patrimonio debe ser igual al valor actual de la compañía en el mercado. Dado que el patrimonio representa un reclamo residual sobre los activos, con pasivos limitados, este se puede modelar como una opción call. La característica de default le permite al tenedor entregar el activo subyacente en forma de pago de la deuda si el valor de este cae por debajo del valor de la deuda. En este caso el activo subyacente es el valor de los activos o valor de la firma. En otras palabras, el accionista puede irse si el valor de la compañía cae por debajo del valor de la deuda. El pago de deuda a su madurez es equivalente a la diferencia entre el valor libre de no pago (libre de default) y una opción put en el valor de la compañía con el strike-price igual al valor en libros de la deuda y su fecha de expiración equivalente a la madurez de la deuda. La opción put representa el reclamo limitado de pasivos suscrito por el tomador de la deuda para con el socio, lo que le da a este último la libertad de someter los activos de la compañía e irse cuando el valor de la mismas caiga por debajo de los pagos prometidos del tomador de la deuda a la madurez.

\section{Metodología}

Gapen, Gray, Hoon Lim y Yingbin, (2006), ilustran la metodología de reclamos contingentes aplicada a un balance simplificado de una compañía comprometida con deuda madura y patrimonio junior. En algún punto del tiempo, el valor total de mercado de los activos A de una compañía financiada con deuda $\mathrm{D}$ y patrimonio $\mathrm{E}$, es igual al valor de mercado del patrimonio más el valor de mercado de la deuda riesgosa. El análisis fundamental establece que el valor de los activos de la compañía se deriva de un proceso estocástico del valor presente descontado de los ingresos menos gastos, con el potencial de que el valor de los activos caiga por debajo del punto en el cual los pagos programados de deuda puedan ser hechos. Si los activos caen por debajo del nivel en el cual la deuda no 
pueda ser servida, el no pago (default) es el resultado. Este nivel es comúnmente conocido como DB (Distress Barrier), y es igual a o cercano al valor de la deuda libre de no pago (libre de default).

Los socios tienen un reclamo contingente junior sobre el valor residual de los activos en el futuro. De este modo, el valor del patrimonio puede ser visto como una opción en la cual los socios reciben el máximo de los activos menos DB, o nada en caso de default. El valor del patrimonio por ende es:

$$
E=\max (A-D B, 0)
$$

Las fórmulas estándar del option- pricing pueden ser usadas para relacionar los cambios en el precio de la compañía con los cambios en el patrimonio. Dada la relación que existe entre el patrimonio de la compañía y sus activos, los cambios en el valor del patrimonio transado pueden ser usados mediante las relaciones del option- pricing para inferir los cambios en el valor del mercado de los activos de la compañía.

Sin embargo, en el caso de la deuda riesgosa todo es un poco más complejo. Los acreedores de la deuda están obligados a absorber las pérdidas en caso de no pago (default) y la garantía de repago por el deudor puede ser modelada como una opción put implícita dado que los acreedores reciben los activos de la compañía que no pague (o de forma equivalente los activos de la compañía son puestos a disposición de los acreedores). Por ende, los acreedores de deuda riesgosa reciben el mínimo del valor libre de default, o en el caso de default, el reclamo maduro en los activos. Desde que el valor de la deuda libre de default es igual al DB y a la opción implícita put de los activos de la compañía (DB-A,0), el valor de la deuda riesgosa puede ser modelado como:

$$
D=D B-\max (D B-A, 0)
$$

Si se insertan estas relaciones de optionpricing a la identidad del balance, resulta en que un valor de mercado de la compañía en un instante de tiempo t de:

$$
\begin{aligned}
& A=D+E \quad y \\
& A=D B-\max (D B-A, 0) \\
& +\max (A-D B, 0)
\end{aligned}
$$

La fórmula de option-pricing es usada en dos pasos. Primero, el valor observado de mercado del patrimonio y el DB son usados con la fórmula de la opción call para derivar el valor de los activos de la compañía. El valor de los activos de la compañía y el DB son usados luego con la fórmula de la opción put para derivar el valor implícito de mercado de la deuda riesgosa. Por ende, el CCA usa las fórmulas put y call del option-pricing" para desarrollar un balance al valor del mercado sobre las variables financieras del mismo.

\subsection{Pasos metodológicos para hacer el cálculo de la estructura óptima de capital}

La metodología utilizada está basada en 
el libro Finanzas corporativas en la práctica de López y Luna (2002).

1. Valorar la deuda hallando el spread de equilibrio que iguala el valor nominal de la deuda y el valor de mercado. Los parámetros mínimos para el cálculo son: a volatilidad del subyacente, la tasa libre de riesgo, los pagos del capital y la política de dividendos.

2. El valor del subyacente, que en este caso es el valor de la empresa, debe ser igual al valor de la empresa con el modelo de FCD y WACC.

3. El FCD requiere el costo de equilibrio de la deuda que se halla en el numeral $1 \mathrm{y}$ el beta operativo que se puede calcular desapalancando el beta o mediante el beta del sector.

Descripción de los parámetros de partida. Uno de los datos centrales es la volatilidad del activo subyacente. Una de las formas tradicionales para hallar la volatilidad es a través del comportamiento de la fluctuación del precio de la acción en el mercado. Dado que esta empresa no cotiza en bolsa, la forma como se calculó este parámetro es mediante la simulación de Montecarlo del FCD. Este parámetro a su vez nos permite hallar los parámetros " $u$ " $y$ " $d$ " con las ecuaciones que se describen en la tabla de parámetros de partida. Igualmente se definen $\mathrm{p}$ y $\mathrm{q}=(1-\mathrm{p})$. El activo subyacente es el valor de la empresa que se calculará por medio de FCD descontado a la tasa WACC. Luego se construyen varios modelos relacionados entre sí mediante una forma iterativa yse presenta el modelo de calendarios de pago de la deuda. Con base en la literatura académica respecto a la proporción de deuda óptima, se tomó el valor de 6.600.000 para la empresa analizada y los términos de pago son intereses periódicos y amortización de la deuda total al final de los cuatro años.

Después de haber precisado los parámetros, se pasa a calcular el valor de la empresa y el valor de la deuda, para tal efecto se utilizará el modelo binomial de valoración de opciones. En cuanto al árbol binomial del valor de la deuda se hacen las siguientes precisiones:

1. Si suponemos que la deuda es única, que tiene prioridad absoluta en caso de quiebra y que no existen costos de liquidación, el valor de la deuda en el último periodo sería el valor menor entre la empresa y el de la deuda (principal más intereses).

2. En los escenarios en que el valor empresarial es superior al valor de deuda más los intereses, el valor de la deuda es precisamente ese, pues el deudor puede hacer frente a sus obligaciones.

3. El modelo matemático para la última rama es el mínimo entre el valor de la empresa en ese periodo por un lado y el valor de los pagos, por el otro.

4. El valor de cada nodo es igual al mínimo de la suma de cada rama que surge del nodo valorado multiplicado por las probabilidades $\mathrm{p}$ y $\mathrm{q}$, y a cada rama se ha descontado $(1+\mathrm{Rf})$, al valor neto se le suma el valor de pago de capital e interés de ese periodo por 
un lado, y por otro lado, el valor de la empresa en ese período.

El paso siguiente es la elaboración del flujo de caja para la valoración de la empresa. Los datos históricos se obtuvieron del sistema de información de riesgo empresarial SIREM, de la Superintendencia de Sociedades.

Ahora se calcula el adjusted present value dentro de la lógica de los desarrollos de MM de 1963. Los pasos que se siguieron consistieron en calcular la siguiente relación funcional del valor de la empresa que está determinada por el valor de la empresa sin apalancamiento financiero $\mathrm{V}(\mathrm{u})$, más el valor presente neto de los ahorros fiscales, menos los costos de quiebra. Los dos primeros datos se calcularon con ecuaciones conocidas. El tercer dato se refiere al diferencial del valor de la empresa calculada por el FCD y el valor de la empresa calculada por el modelo APV.

Para el cálculo de la estructura óptima se procede así:

1. Se calcula el valor de la deuda del mercado mediante el modelo de opciones desarrollado por Merton en 1974.

2. En segundo término, se encuentra $e l$ spread de equilibrio con un proceso de iteración. En nuestro caso se elaboró con la función objetivo. La pretensión es igualar el valor de la deuda de mercado y el valor nominal de la deuda y despejar así el valor de spread de default. En concreto, se halla el costo de la deuda como una sumatoria de una tasa libre de riesgo y un spread por probabilidad de default. Este spread está determinado por la volatilidad del activo subyacente y por las condiciones del pago del crédito de forma central.

3. Como se dijo antes, se hizo el FCD con unos value driver históricos y se supuso que la empresa se comportará como lo viene haciendo.

4. Posteriormente se hizo una tabla de parámetros dentro de los lineamientos de CAPM con los supuestos que se han comentado. Al analizar la tabla correspondiente debe quedar claro que hay una circularidad en cuanto al valor de la empresa. Se parte de un estimativo de este valor y el resultado es este mismo concepto pero recalculado.

5. En cuanto a la tabla de trade-off, lo que se busca es hallar los valores del modelo como es el valor marginal de los costos de quiebra y los costos marginales de los ahorros fiscales. En el momento que se igualan estos dos valores, se define en términos de la literatura que no sería conveniente una mayor contratación de deuda porque es el mayor valor de la empresa que, a su vez, está asociado a una arquitectura óptima de fuentes de financiación.

\section{Resultados}

\subsection{Estructura financiera de la empresa Hilanderías Bogotá S.A.}

Hilanderías Bogotá S.A. es una 
empresa colombiana dedicada a la fabricación de hilazas $100 \%$ acrílicas, mezclas con fibras naturales y artificiales, lycra recubierta, cotton, angora, cashmere y lurex look entre otras hilazas. Los productos se utilizan como materia prima en la industria textil de tejido de punto, tejido plano, calcetería, artículos decorativos y artesanales, entre otros. Hilanderías Bogotá S.A. fue constituida con capital netamente colombiano, el 30 de noviembre de 1965, con el objeto de cubrir el déficit de hilazas acrílicas en el mercado colombiano.

En un principio, la Empresa se dedicó a la importación de materias primas para su procesamiento en las hilanderías existentes y en 1967 inició el programa industrial con la construcción de los $10.000 \mathrm{~m}^{2}$ de las actuales instalaciones en Bosa (Bogotá). Hilanderías Bogotá S.A. se ha caracterizado por la inversión permanente en maquinaria y equipo con tecnología de punta para lograr la fabricación de hilazas de la más alta calidad. Sus inversiones tecnológicas se han enfocado hacia la innovación permanente de procesos y productos que permitan a sus clientes desarrollar prendas de alta calidad y variado diseño.

Los balances de la empresa se encuentran en miles de pesos y los cálculos que se desprenden de ellos).

\subsubsection{Evolución del pasivo y del patrimonio}

En general, el comportamiento del pasivo no tiene una tendencia marcada de crecimiento o decrecimiento sino que ha sido intercalado (un año aumenta, otro año disminuye) combinando la financiación entre obligaciones financieras a corto plazo y proveedores principalmente y algunas veces con reservas y utilidades. Respecto al patrimonio ha tenido un comportamiento creciente, especialmente a causa del crecimiento de sus reservas. Podríamos decir que la empresa se financia básicamente con recursos externos a corto plazo.

\subsubsection{Composición del pasivo}

El pasivo está compuesto principalmente por proveedores en un 50,72\% y obligaciones financieras a corto plazo en un $26,24 \%$, seguida de cuentas por pagar en $11,61 \%$, los impuestos, gravámenes y tasas y obligaciones laborales de corto plazo participan cerca de un $5 \%$. En general, se observa una tendencia a disminuir las obligaciones a corto plazo por proveedores y cuentas por pagar.

\subsubsection{Composición de la deuda financiera por plazo}

La composición de la deuda por plazo en el sector está compuesta en un 91,53\% por el corto plazo y en $8,47 \%$ por largo plazo, observándose una tendencia a usar en un $100 \%$ solo deuda a corto plazo. 
Pudiéndose apreciar que la preferencia por la financiación a corto plazo se da por causa de su costo más bajo y requiere menos trámites para su consecución.

En general, las principales fuentes de financiamiento de la empresa Hilanderías Bogotá S.A. son los recursos externos, utiliza las obligaciones a corto plazo, proveedores y cuentas por pagar de forma intercalada, observándose una tendencia a utilizar en una proporción mayor a los proveedores. La empresa no utiliza fuentes de financiamiento a largo plazo ni recursos internos.

\subsection{Determinación de la estructura óptima de capital}

En el Apéndice A se encuentran todas las tablas que contienen los cálculos necesarios para determinar la estructura óptima de capital. La estructura óptima de capital para la empresa Hilanderías Bogotá S.A. se halla entre el intervalo $53 \%$ y $54 \%$ de proporción de deuda.

\section{Conclusiones}

1. Las principales fuentes de financiamiento de la empresa Hilanderías Bogotá S.A son los recursos externos. Utilizando las obligaciones de corto plazo, proveedores y cuentas por pagar de forma intercalada, observándose una tendencia a emplear en una proporción mayor a los proveedores. La empresa no utiliza fuentes de financiamiento a largo plazo ni recursos internos.
2. De acuerdo con los resultados del modelo en la Tabla 4.3.1, se reafirma: - El costo de Ke aumenta en la medida de la contratación adicional de deuda. - El costo de la deuda $\mathrm{Kb}$ disminuye en la parte izquierda de la relación óptima de deuda-capital, y después se incrementa.

- Aquí se comprueba que el WACC encuentra un punto mínimo donde se encuentra el punto óptimo de $\mathrm{D} / \mathrm{E}$.

- El modelo permite calcular costos de quiebra.

- El modelo permite calcular los el ahorro fiscal.

- El modelo determinó la estructura óptima de capital.

3. El modelo presenta cómo se pueden analizar las relaciones entre accionistas y acreedores a partir de la teoría de opciones, asimilándose el patrimonio a una opción call y la deuda a una opción put. Las condiciones que los acreedores imponen a los accionistas son reflejadas en la valoración de deuda.

4. El desafío de otras investigaciones referentes al tema es introducir la modelación en ambientes de incertidumbre. Es decir, incorporar en la modelación la sensibilidad del modelo a choques externos por cambios macroeconómicos, cambios de tendencia de la estructura de mercado, cambios tecnológicos, que puedan ser significativos en la generación de valor. No obstante se 
admite que el modelo reconoce un tipo de riesgo como el sistemático (beta) y la volatilidad del subyacente que es un valor único. La gerencia moderna permite implementar políticas de mitigación y de transferencia de riesgo para empresas que están pocos diversificadas.

\section{R eferencias bibliográficas}

1. Banco de la República.(2005).Estructura financiera del sector corporativo privado. Reportes del Emisor, $N^{\circ} 76$.

2. Chacón, O., Arroyo, Y.\& Villaba, M.(2007).Teoría del trade-off para la definición de la estructura de financiación de las PYME's de Bucaramanga.Revista de la Facultad de Ingenierías Físico Mecánicas,Universidad Industrial de Santander, 6(2).

3. Cruz, J.\& Wadnipar, S.(2008).Análisis de variables y modelos que intervienen en la determinación de la estructura de capital de las empresas colombianas. Working paperCESA. Recuperado dehttp://repository.cesa.edu.co/handle/ 10726/111

4. Deangelo, H.\&Masulis, R.(1980). Leverage and dividend irrelevancy under corporate and personal taxation.The Journal of Finance, 35 (2).

5. Durand, D. (1959). The cost of capital, corporation finance, and the theory of investment:comment.American Economic Review, 49, (4).

6. Fama, E. \& French, K. (2002).Testing trade-off and pecking order predictions about dividends and debt.The Review of Financial Studies, 15 (1).

7. Gilson, S. (1997).Transactions costs and capital structure choice: evidence from financially distressed firms. The Journal of Finance, 5 (1).

8. Gapen, M., Gray, D.,Cheng, H.\&Yingbin, X.(2006). The contingent claims approach to corporate vulnerability analysis: estimating default risk and economy wide risk transfer.International Monetary Fund, WP/04/121.

9. Harris, M.\&Raviv, A.(1991).The theory of capital structure.The Journal of Finance, 46 (1).

10. Hilanderías Bogotá. (s.f.). Recuperado de http://www.hilanderiasbogota.com/ Acerca_de_Nosotros/acerca.htm

11. Jensen, M. y M, W. (1976). Theory of the firm: managerial behavior, agency costs, and ownwerhip structure.En Journal of financialeconomics, No 3. Recuperado de: http://hupress.harvard.edu/ catalog/JENTHF.html.

12. López, F. \& Luna, W.(2002).Finanzas corporativas en la práctica.(1ra. ed. en 
español). Madrid: Mc Graw Hill, Interamericana de España, S.A.U.

13. Miller, M.(1977). Debt and taxes.The Journal of Finance, 32 (2).

14. Modigliani, F. \&Miller, M. (1958).The cost of capital, and H. Miller Corporation finance and the theory of investment. The American Economic Review, 48(3).

15. Modigliani, F. \&Miller.M. (1959).The cost of capital, corporation finance, and the theory of investment: reply.The American Economic Review, 49(4).

16. Modigliani, F. \&Miller, M. (1963).Corporate income taxes and the cost of capital: a correction.The American Economic Review, 53(3).

17. Myers, S. \& Majluf, N. (1983). Corporatefinancing and investment decisions when firms have information that investors do not have. Journal of FinancialEconomics, 13.

18. Rivera, J.(2007). Estructura financiera y factores determinantes de la estructura de capital de las PYMES del sector de confecciones del Valle del Cauca en el período 2000-2004.Cuadernos de Administración, Universidad del Valle, 20(34).
19. Rivera, J.(2002). Teoría sobre la estructura de capital. Estudios Gerenciales (84).

20. Ryan, B., Scapens, R. \& Theobald, M.(2004). Metodología de la investigación en finanzas y contabilidad. Barcelona: Deusto.

21. Sarmiento, R.(2005).La estructura de financiamiento de las empresas: una evidencia teórica y econométrica para Colombia. 1997-2004. Documentos de Economía,Facultad de Ciencias Económicas y Administrativas, Pontificia Universidad Javeriana (16).

22. Stulz, R.(1990).Managerial discretion and optimal financing policies.Journal of Financial Economics, 26.

23. Tenjo, F., López, E. \&Zamudio, N.(2006).Determinantes de la estructura de capital de las empresas.Banco de la República, Borradores de Economía (380). Recuperado de http://http:// www.banrep.gov.co/docum/ftp/ borra380.pdf

24. Wessels, R. \& Titman, S. (1988). The determinants of capital structure choice. The Journal of Finance, 43, (1). 
A PUNTES DEL CENES N $N .52$

Vol. 30, Segundo Semestre de 2011

\section{APÉNDICE A}

Tabla 1. Parámetros iniciales

\begin{tabular}{|l|r|l|l|}
\hline \multicolumn{1}{|c|}{ Volatilidad Anual } & $25 \%$ & Dato que se calculó mediante una simulación Montecarlo \\
\hline $\mathrm{h}$ & 1 & & \\
\hline $\mathrm{u}$ & 1,284 & $\mathrm{u}$ se halla como & $u$ e $\sqrt{t / n}$ \\
\hline $\mathrm{d}$ & 0,779 & d se halla como & $\mathrm{d}=1 / \mathrm{u}$ \\
\hline $\mathrm{Rf}$ & $4,25 \%$ & Dato dado & $p \quad \frac{\hat{r}}{u} d$ \\
\hline $\mathrm{p}$ & $52,2 \%$ & $\mathrm{p}$ se halla como & \\
\hline $\mathrm{q}$ & $47,8 \%$ & q se halla como & $q=1 / p$ \\
\hline $\mathrm{S}$ El valor de la empresa & $12.117 .966,6$ & Viene del modelo de valoración \\
\hline Nominal de la deuda & 6.600 .000 & Tomado de balance como deuda financiera & \\
\hline Valor mercado del patrim. & $5.517 .966,6$ & valor de la empresa - valor deuda & \\
\hline Spread & 0,00914000 & Valor que se obtiene de iteración. & \\
\hline Tasa interés & $5,16400 \%$ & Dato dado & \\
\hline
\end{tabular}

E $\quad=$ valor a precio de mercado de los recursos propios.

$\mathrm{D} \quad=$ valor a precio de mercado de la deuda.

$\mathrm{V}=\mathrm{E}+\mathrm{D}$

$\mathrm{Ke}, 1=$ coste de los recursos propios, para el nivel de endeudamiento correspondiente.

$\mathrm{Kd}=$ coste de la deuda.

$\mathrm{T}=$ tasa impositiva.

WACC $=$ coste medio ponderado de capital.

$\mathrm{Rf} \quad=$ rentabilidad libre de riesgo.

$\mathrm{PM}=$ prima de mercado.

$\mathrm{Be}, 1=$ beta apalancada.

$\mathrm{Ke}, \mathrm{u}=$ costo del $\mathrm{E}$ si no existe deuda.

$\mathrm{Be}, \mathrm{u}=$ beta desapalancada. 
La estructura óptima de capital con reclamos contingentes...

M aría del Pilar Báez Roa - J uan Sergio Cruz M erchán

Tabla 2. Calendarios de pagos de la deuda

\begin{tabular}{|c|c|c|c|c|c|}
\hline Años & 1 & 2 & 3 & 4 & 5 \\
\hline \multicolumn{6}{|l|}{ Deuda } \\
\hline Intereses & $340.824,0$ & $340.824,0$ & $340.824,0$ & $340.824,0$ & \multirow{10}{*}{$0,0 \%$} \\
\hline Amort. capital & & - & - & $6.600 .000,0$ & \\
\hline Saldo deuda & $6.600 .000,0$ & $6.600 .000,0$ & $6.600 .000,0$ & & \\
\hline Total pagos & $340.824,00$ & $340.824,00$ & $340.824,00$ & $6.940 .824,00$ & \\
\hline Dividendos & - & - & & & \\
\hline Total pagos & $340.824,00$ & $340.824,00$ & $340.824,00$ & $6.940 .824,00$ & \\
\hline Ratio Div/S & $0,0 \%$ & $0,0 \%$ & $0,0 \%$ & $0,0 \%$ & \\
\hline valor deuda & 528547 & & & & \\
\hline Vr actual pagos & $324.088,1$ & $308.174,0$ & $293.041,3$ & $5.674 .696,6$ & \\
\hline \multirow[t]{2}{*}{ Vr actual pagos xt } & $324.088,1$ & $616.348,0$ & $879.124,0$ & $22.698 .786,4$ & \\
\hline & & & & Duración & 3,71 \\
\hline
\end{tabular}

Tabla 3. Valor de la empresa

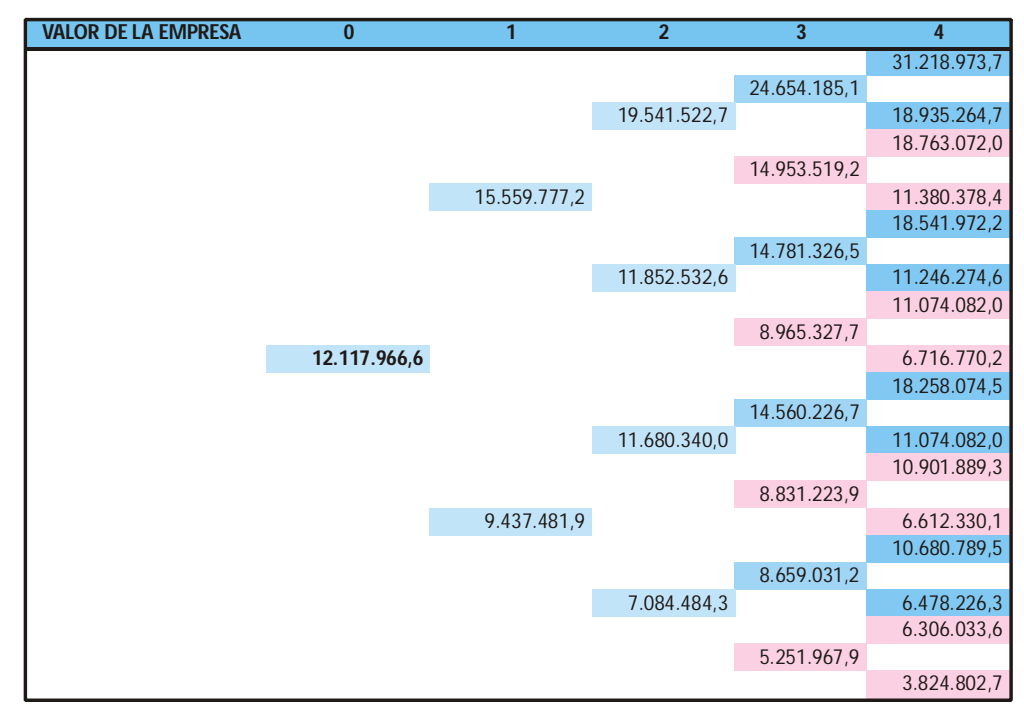


A PU N TES DEL CEN ES N 0.52

Vol. 30, Segundo Semestre de 2011

Tabla 4. Valor de la deuda

\begin{tabular}{|c|c|c|c|c|c|}
\hline VALOR DE LA DEUDA & 0 & 1 & 2 & 3 & 4 \\
\hline & \multirow{17}{*}{$\begin{array}{l}\mathbf{5 . 5 1 7 . 7 2 4 , 9} \\
6.600 .241,7\end{array}$} & & & & $6.940 .824,0$ \\
\hline & & & & $6.998 .688,7$ & \\
\hline & & & $7.054 .194,5$ & & $\begin{array}{l}6.940 .824,0 \\
6.940 .824,0\end{array}$ \\
\hline & & $8.473 .945,0$ & & $6.998 .688,7$ & \\
\hline & & $7.085 .832,2$ & & & $\begin{array}{l}6.940 .824,0 \\
6.940 .824,0\end{array}$ \\
\hline & & \multirow{12}{*}{$\begin{array}{l}\mathbf{2 . 7 8 0 . 6 3 7 , 9} \\
6.656 .844,0\end{array}$} & & $6.998 .688,7$ & \\
\hline & & & $7.007 .079,8$ & & $\begin{array}{l}6.940 .824,0 \\
6.940 .824,0\end{array}$ \\
\hline & & & & $6.895 .945,2$ & \\
\hline & & & & & $\begin{array}{l}6.716 .770,2 \\
6.940 .824,0\end{array}$ \\
\hline & & & & $6.998 .688,7$ & \\
\hline & & & $6.985 .117,8$ & & $\begin{array}{l}6.940 .824,0 \\
6.940 .824,0\end{array}$ \\
\hline & & & & $6.848 .052,5$ & \\
\hline & & & & & $\begin{array}{l}6.612 .330,1 \\
6.940 .824,0\end{array}$ \\
\hline & & & & $6.786 .557,0$ & \\
\hline & & & $6.146 .999,8$ & & $\begin{array}{l}6.478 .226,3 \\
6.306 .033,6\end{array}$ \\
\hline & & & & $5.251 .967,9$ & \\
\hline & & & & & $3.824 .802,7$ \\
\hline
\end{tabular}

Tabla 5. Valoración de la deuda por medio de FCD

\begin{tabular}{|c|c|c|c|c|c|c|}
\hline AÑO & & 1 & 2 & 3 & 4 & 5 \\
\hline Ingresos & & 18.752 .658 & 18.752 .658 & 18.752 .658 & 18.752 .658 & 18.752 .658 \\
\hline Incremento ingresos & & 0,02 & 0,02 & 0,02 & 0,02 & 0,02 \\
\hline Costos operativos & 0,937974499 & 17.589 .515 & 17.589 .515 & 17.589 .515 & 17.589 .515 & 17.589 .515 \\
\hline Amortización & $1 \%$ & 187.527 & 187.527 & 187.527 & 187.527 & 187.527 \\
\hline Provisiones & 0,00500 & 93.763 & 93.763 & 93.763 & 93.763 & 93.763 \\
\hline BAIT & & 881.853 & 881.853 & 881.853 & 881.853 & 881.853 \\
\hline Intereses & & 340.824 & 340.824 & 340.824 & 340.824 & 0 \\
\hline BAT & & 541.029 & 541.029 & 541.029 & 541.029 & 881.853 \\
\hline \multicolumn{7}{|l|}{ Base negativa del año } \\
\hline \multicolumn{7}{|l|}{ Base negativas acumuladas } \\
\hline \multicolumn{7}{|l|}{ Base negativas aplicadas } \\
\hline Base impositiva & & 541.029 & 541.029 & 541.029 & 541.029 & 881.853 \\
\hline Impuesto & $35 \%$ & 189.360 & 189.360 & 189.360 & 189.360 & 308.649 \\
\hline Tipo impositivo efectivo & & 0,350 & 0,350 & 0,350 & 0,350 & 0,350 \\
\hline Beneficio Neto & & 351.669 & 351.669 & 351.669 & 351.669 & 573.205 \\
\hline FGO & & 539.196 & 539.196 & 539.196 & 539.196 & 760.731 \\
\hline Recuperación provisiones & & 0 & 0 & 0 & 0 & 0 \\
\hline BAIT(1-T) & & 573.205 & 573.205 & 573.205 & 573.205 & 573.205 \\
\hline Amortización & & 187.527 & 187.527 & 187.527 & 187.527 & 187.527 \\
\hline Provisiones & & 93.763 & 93.763 & 93.763 & 93.763 & 93.763 \\
\hline Recuperación provisiones & & 0 & 0 & 0 & 0 & 0 \\
\hline Inversiones & & 15.000 & 15.000 & 15.000 & 15.000 & 15.000 \\
\hline FCL & & 651.968 & 651.968 & 651.968 & 651.968 & 651.968 \\
\hline Pagos deuda & & 0 & 0 & 0 & 6.600 .000 & 0 \\
\hline Saldo deuda & & 6.600 .000 & 6.600 .000 & 6.600 .000 & 0 & 0 \\
\hline FCA & & 430.432 & 430.432 & 430.432 & -6.169 .568 & 651.968 \\
\hline
\end{tabular}


La estructura óptima de capital con reclamos contingentes...

M aría del Pilar Báez Roa - J uan Sergio Cruz M erchán

Tabla 6. Valoración de la empresa por medio de FCD

\begin{tabular}{|c|c|c|c|c|c|c|}
\hline \multicolumn{7}{|l|}{ VALORACION } \\
\hline AÑo & & 1 & 2 & 3 & 4 & 5 \\
\hline Factor descuento deuda & & 1,05 & 1,11 & 1,16 & 1,22 & 1,29 \\
\hline Valor pagos deuda & 6.600 .000 & 324.088 & 308.174 & 293.041 & 5.674 .697 & 0 \\
\hline Valor de mercado $\mathrm{E}$ & 5.517 .967 & 5.574 .012 & 5.634 .039 & 5.698 .331 & 12.278 .179 & 12.510 .240 \\
\hline Valor de mercado D & 6.600 .000 & 6.600 .000 & 6.600 .000 & 6.600 .000 & 0 & 0 \\
\hline$D / E$ & 1,20 & 1,18 & 1,17 & 1,16 & 0,00 & 0,00 \\
\hline$E /(E+D)$ & 0,5 & 0,46 & 0,46 & 0,46 & 1,00 & 1,00 \\
\hline$D /(E+D)$ & 0,5 & 0,54 & 0,54 & 0,54 & 0,00 & 0,00 \\
\hline $\mathrm{Kd}$ & & 0,0516 & 0,0516 & 0,0516 & 0,0516 & 0,0516 \\
\hline Kd di & & 0,03 & 0,03 & 0,03 & 0,03 & 0,03 \\
\hline Rf & & 0,05 & 0,05 & 0,05 & 0,05 & 0,05 \\
\hline Prima mercado & & 0,04 & 0,04 & 0,04 & 0,04 & 0,04 \\
\hline Beta desapalancado & & 0,60 & 0,60 & 0,60 & 0,60 & 0,60 \\
\hline Keu & & 0,07 & 0,07 & 0,07 & 0,07 & 0,07 \\
\hline Beta apalancado & 1,07 & 1,06 & 1,06 & 1,05 & 0,60 & 0,60 \\
\hline Beta de la deuda & & 0,02 & 0,02 & 0,02 & 0,02 & 0,02 \\
\hline Beta deuda neta de Imp. & & $-0,50$ & $-0,50$ & $-0,50$ & $-0,50$ & $-0,50$ \\
\hline Kel & & 0,0882 & 0,0880 & 0,0878 & 0,0720 & 0,0720 \\
\hline WACC & & 0,06 & 0,06 & 0,06 & 0,07 & 0,07 \\
\hline Factor descuento de Ke & & 1,09 & 1,18 & 1,29 & 1,38 & 1,48 \\
\hline Factor descuento de WACC & & 1,06 & 1,12 & 1,19 & 1,27 & 1,36 \\
\hline VA CFA & $(2.934 .905,0)$ & $395.558,77$ & $363.568,29$ & $334.220,43$ & $-4.468 .771,70$ & $440.519,16$ \\
\hline VA FCF wacc & $12.117 .966,6$ & 615.899 & 581.789 & 549.531 & 512.623 & 9.858 .126 \\
\hline Deuda & $6.600 .000,0$ & & & & & \\
\hline Recursos propios & $5.517 .966,6$ & & & & & \\
\hline
\end{tabular}

Tabla 7. Valoración APV

\begin{tabular}{|l|r|r|r|r|r|r|}
\hline VALORACIÓN APV & & \multicolumn{1}{|c|}{$\mathbf{1}$} & \multicolumn{1}{|c|}{$\mathbf{2}$} & \multicolumn{1}{|c|}{$\mathbf{3}$} & \multicolumn{1}{|c|}{$\mathbf{5}$} & 1,23 \\
\hline Factor Descuento Keu & & 1,07 & 1,15 & 52 & 1,42 \\
VA FCL & $11.692 .297,2$ & 608.179 & 567.331 & 529.227 & 493.682 & 9.493 .879 \\
D x T x Keu & & 187.152 & 186.354 & 184.956 & 183.498 & 0 \\
Va de los ahorros fiscales & $625.827,4$ & 174.582 & 162.162 & 150.136 & 138.948 & \\
Valor empresa & $12.318 .124,6$ & & & & & \\
Costo de quiebra & $200.157,9$ & & & & \\
\hline
\end{tabular}

Tabla 8. Determinación de la estructura óptima de capital

\begin{tabular}{|c|c|c|c|c|c|c|c|c|c|c|c|}
\hline $\begin{array}{c}\text { Valor } \\
\mathbf{D}\end{array}$ & $\begin{array}{c}\text { Valor } \\
\mathbf{E}\end{array}$ & $\mathbf{D} / \mathbf{( D + E )}$ & $\begin{array}{c}\mathbf{V} \\
\text { Empresa }\end{array}$ & $\mathbf{K d}$ & $\mathbf{K e}$ & $\mathbf{W A C C}$ & $\begin{array}{c}\text { Costo de } \\
\text { quiebra }\end{array}$ & $\begin{array}{c}\text { Incremento } \\
\text { quiebra }\end{array}$ & $\begin{array}{c}\text { Ahorro } \\
\text { Fiscal }\end{array}$ & $\begin{array}{c}\text { Incremento } \\
\text { AF }\end{array}$ & $\begin{array}{c}\text { Difer } \\
\text { Increm }\end{array}$ \\
\hline 5.900 .000 & 6.217 .107 & $49 \%$ & 12.117 .107 & 0,04720 & 0,0848 & 0,0585 & 127.349 & & & 552.159 & \\
6.200 .000 & 5.933 .237 & $51 \%$ & 12.133 .237 & 0,04774 & 0,0861 & 0,0580 & 140.285 & 12.936 & 581.225 & 29.066 & 16.130 \\
6.400 .000 & 5.734 .483 & $53 \%$ & 12.134 .483 & 0,04894 & 0,0871 & 0,0579 & 160.059 & 19.774 & 602.246 & 21.020 & 1.246 \\
6.600 .000 & 5.517 .967 & $54 \%$ & 12.117 .967 & 0,0516 & 0,08816251 & 0,058426746 & 200.158 & 40.099 & 625.827 & 23.582 & -16.517 \\
\hline
\end{tabular}

